\title{
Correction: Obesity without metabolic disorder and silent brain infarcts in a neurologically healthy population
}

Ki-Woong Nam · Hyung-Min Kwon (ib) - Han-Yeong Jeong • Jin-Ho Park • Hyuktae Kwon $(\mathbb{D} \cdot$ Su-Min Jeong

Published online: 17 February 2020

(c) Springer Nature Limited 2020

\section{Correction to: International Journal of Obesity} https://doi.org/10.1038/s41366-019-0372-6 into "aneurologically." This has been corrected in the PDF and HTML versions of this article.

This article was originally published with an erroneous title; the words "a" and "neurologically" were incorrectly merged 